Z. klin. Chem. u. klin. Biochem.

7. Jg., S. 175-178, März 1969

\title{
Enzymaktivitäten und Proteinsynthese in geschädigten Geweben
}

\author{
Von H. KRöNER und W. StaIB \\ Aus dem Institut für Physiologische Chemie der Universität Düsseldorf
}

(Eingegangen am 21. Mai 1968)

Kurzfristig nach intraperitonealer Injektion von Tetrachlorkohlenstoff ist der Einbau von Leucin-[1-14 C] in das Leberprotein in vivo deutlich gehemmt. Der Einbau von Orotsäure- $\left[5-{ }^{3} \mathrm{H}\right]$ in die RNA unter den gleichen Bedingungen ist nicht verändert. Eine gesteigerte Neusynthese von Enzymprotein kann damit nicht die Ursache für die früher beobachtete Aktivitätszunahme von Leberenzymen nach Tetrachlorkohlenstoff sein. Auch bei aufgehobener Proteinsynthese während totaler Ischämie findet sich eine Aktivitätszunahme zahlreicher Enzyme in Leber und Herzmuskel. Die Ursache dieses Enzymanstieges und seine Bedeutung für die Freisetzung von Serumenzymen aus geschädigten Geweben wird kurz diskutiert.

\section{Enzyme activities and protein synthesis in damaged livers}

Shortly after the intraperitoneal injection of carbon tetrachloride, the incorporation of $\left[1-{ }^{14} \mathrm{C}\right]$ leucine into liver protein in vivo is markedly inhibited. Under the same conditions the incorporation of $\left[5-{ }^{3} \mathrm{H}\right]$ orotic acid into RNA is not altered. An increased de novo synthesis of enzyme protein cannot therefore account for the previously observed increase in the activity of liver enzymes following treatment with carbon tetrachloride. Even when protein synthesis is abolished during total ischaemia, there is an incraese in the activity of many enzymes in liver and heart muscle. The reason for this enzyme increase and its connection with the release of serum enzymes from damaged tissues is briefly discussed.

In einer früheren Untersuchung (1) konnten wir zeigen, $\mathrm{da} ß$ in den ersten Stunden einer Tetrachlorkohlenstoffvergiftung bei der Ratte die Aktivität verschiedener Leberenzyme ansteigt. Es handelt sich um die gleichen Enzyme, deren Aktivität bei einer Leberschädigung durch Tetrachlorkohlenstoff $(2,3)$ oder auf Grund einer Hepatitis (4) auch im Serum zunimmt, wie die Lactatdehydrogenase (EC 1.1.1.27) (LDH), Malatdehydrogenase (EC 1.1.1.37) (MDH), Sorbitdehydrogenase (L-Iditdehydrogenase, EC 1.1.1.14) (SDH), Glutamatdehydrogenase (EC 1.4.1.2) (GIDH), Alanintransaminase (EC 2.6.1.2) (GPT) und Aspartattransaminase (EC 2.6.1.1) (GOT). Die Aktivitätszunahme der Enzyme in der Leber erscheint uns in doppelter Hinsicht interessant, einmal bezüglich des recht spezifischen Wirkungsmechanismus des Tetrachlorkohlenstoffes auf die Leber, zum anderen bezüglich der Freisetzung von Serumenzymen bei Zellschädigungen ganz allgemein.

$\mathrm{Da}$ uns eine einfache Aktivierung so unterschiedlicher Enzyme als Ursache für die Zunahme der Aktivität unwahrscheinlich erschien, haben wir in der vorliegenden Untersuchung zunächst die Proteinsynthese und gleichzeitig die RNA-Synthese nach Gabe von $\mathrm{CCl}_{4}$ analysiert, und zwar über den Einbau von markiertem Leucin bzw. markierter Orotsäure in vivo. Auf Grund der Ergebnisse ist eine Enzymneusynthese als Ursache der Aktivitätszunahme höchst unwahrscheinlich. Damit lag es nahe, Gewebsschädigungen durch absolute Ischämie zu untersuchen, die einerseits zum Anstieg von Serumenzymen führen, bei denen andererseits eine Proteinneusynthese von vornherein auszuschließen ist. Nach einer durch Inkubation in vitro erzeugten Ischämie haben wir in Leber und Herzmuskel die eingangs aufgezählten Enzyme untersucht, im Herzmuskel zu- sätzlich die Kreatinphosphokinase (EC 2.7.3.2) (CPK). Dabei konnten wir ein ähnliches Verhalten dieser Enzyme wie bei der Tetrachlorkohlenstoffvergiftung in der Leber feststellen.

\section{Methodik}

Männliche Wistar-Ratten (Firma Brünger, Bokel), 200-300 g schwer, erhielten bis zum Versuchsbeginn Standardfutter der Firma Höveler, Langenfeld-Immigrath. Tetrachlorkohlenstoff p. a. (Firma E. Merck), wurde $1 \mathrm{ml} / \mathrm{kg}$ zusammen mit dem vierfàchen Volumen Olivenöl intraperitoneal injiziert. Danach bekamen die Tiere nur noch Trinkwasser. $30 \mathrm{Min}$. vor der Tötung erhielten die Tiere eine intraperitoneale Injektion von Leucin$[1-14 \mathrm{C}], 20 \mu \mathrm{C} / \mathrm{kg}$ und Orotsäure-[5-3 $\mathrm{H}], 60 \mu \mathrm{C} / \mathrm{kg}$. Das Leucin hatte eine spezifische Aktivität von $27,5 \mu \mathrm{C} / \mathrm{mg}$, die Orotsäure eine solche von $5000 \mathrm{C} / \mathrm{Mol}$. In leichter Äthernarkose wurde mittels Frierstopp ein Stück Leber entnommen, anschließend wurden die Tiere getötet.

Aus der zerkleinerten Leber wurden, wie früher beschrieben (5), mit kalter Perchlorsäure die niedermolekularen Bestandteile extrahiert (Extrakt I) und nach Behandlung des Rückstandes mit 1N NaOH die teilhydrolysierte RNA ebenfalls mit kalter Perchlorsäure extrahiert (Extrakt II). Der Rückstand enthält dann das Protein und die DNA.

Die Proteinbestimmung erfolgte mit dem Biuretreagenz nach Weicriselbaum (6), Eichsubstanz war Serumalbumin vom Rind (Behringwerke, Marburg). Die RNA wurde durch Extinktionsmessung bei $260 \mathrm{~nm}$ bestimmt, die $D N A$ wurde nach Burton (7) bestimmt. Als Eichsubstanz diente in beiden Fällen hydrolysiertc Kalbsthymus-DNA (Serva, Heidelberg).

Die Messung der Radioaktivität erfolgte im Tri-Carb Liquid-Scintillation Counter der Firma Packard Instruments, Quenchkorrektur erfolgte mit externem Standard. Gemessen wurde die ${ }^{14} \mathrm{C}$-Aktivität des Leucin im Protein und im Extrakt I, ferner die Tritium-Aktivität der Orotsäure in der teilhydrolysierten RNA (Extrakt II) und im Extrakt I (Einzelheiten siehe 1. c. (8)).

Für die Enzymaktivitätsmessungen nach "Ischämie“ wurden normal ernährte Tiere in Xthernarkose durch Eröffnen der Bauchaorta entblutet, Leber oder Herz ganz entnommen und bei $37^{\circ}$ in feuchter Kammer inkubiert. Von den Lebern wurde vor der Inkubation und nach verschiedenen Inkubationszeiten ein Stück 
Tab. 1

Protein-, RNA- und DNA-gehalt der Rattenleber, sowie Einbauraten von Leucin-[1-1"C] und Orotsăure-[5-2H] in die Leber in vivo nach Gabe von $\mathrm{CCl}_{3}, 1 \mathrm{ml} / \mathrm{kg}$ i. p. Mittelwerte von je 5 Tieren \pm Standardabweichung. Einzelheiten siehe Methodik

\begin{tabular}{|c|c|c|c|c|}
\hline \multirow{2}{*}{ 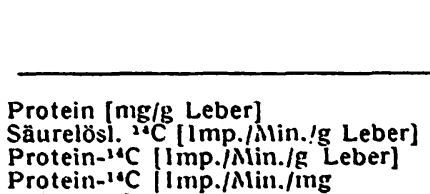 } & \multicolumn{2}{|c|}{$\begin{array}{l}1 \text { Std. nach Injektion } \\
\text { Kontrollen }\end{array}$} & \multicolumn{2}{|c|}{$\begin{array}{l}2 \text { Stdn. nach Injektion } \\
\text { Kontrollen }\end{array}$} \\
\hline & $\begin{array}{c}219 \pm 7 \\
6491 \pm 538 \\
29400 \pm 2750\end{array}$ & $\begin{aligned} 221 & \pm 14 \\
7884 & \pm 565^{*} \\
18800 & \pm 3250^{* * *}\end{aligned}$ & $\begin{array}{c}199 \pm 8 \\
6936 \pm 319 \\
33100 \pm 1505\end{array}$ & $\begin{array}{r}204 \pm 12 \\
10870 \pm 1340 * * \\
16500 \pm 5210^{* * *}\end{array}$ \\
\hline $\begin{array}{l}\text { Protein-"C IImp./Min./mg } \\
\text { Protein] } \\
\text { RNA [mg/g Leber] } \\
\text { Saurelösl. 'H [Imp./Min./g Leber] } \\
\text { RNA-'H [Imp./Mlin./g Leber] } \\
\text { RNA-2H [Imp./Mlin./mg RNA] } \\
\text { DNA [mg/g Leber] }\end{array}$ & $\begin{aligned} 134 & \pm 17 \\
11,7 & \pm 0,3 \\
85200 & \pm 6190 \\
6530 & \pm 812 \\
558 & \pm 62 \\
2,14 & \pm 0,15\end{aligned}$ & $\begin{array}{c}86 \pm 13^{* *} \\
11,9 \pm 0,5 \\
83500 \pm 8270 \\
7580 \pm 1130 \\
635 \pm 78 \\
2,34 \pm 0,14\end{array}$ & $\begin{aligned} & 166 \pm 10 \\
& 11,0 \pm 0,1 \\
& 86600 \pm 1480 \\
& 6475 \pm 535 \\
& 592 \pm 41 \\
& 2,22 \pm 0,17\end{aligned}$ & $\begin{aligned} & 91 \pm 22 * * \\
& 11,0 \pm 0,3 \\
& 79700 \pm 8700 \\
& 7440 \pm 980 \\
& 677 \pm 87 \\
& 2,22 \pm 0,24\end{aligned}$ \\
\hline
\end{tabular}

$* \mathrm{p}<0,01 ; \quad * * \mathrm{p}<0,001$.

entnommen und homogenisiert. Bein Herzen wurde nach 4 Stdn. Inkubation das ganze Organ homogenisiert, nicht inkubierte Herzen dienten als Kontrollen. Die Lebern wurden teils mit bidest. W/asser, teils mit 0,1.N Phosphatpuffer $\mathrm{pH} 7,5$, die Herzen mit Phosphatpuffer im MSE-Homogenisator zerkleinert. Dic Homogenate wurden $30 \mathrm{Min}$. bei $40000 \mathrm{~g}$ in der Kühlzentrifuge zentrifugiert. Im Uberstand wurden folgende Enzyme gemessen: Lactatdehydrogenase, Malatdehydrogenase, Aspartattransaminase, in der Leber zusätzlich Sorbitdehydrogenase und Glutamatdehydrogenase, in Herzmuskel zusätzlich Kreatinphosphokinase. Dic Bestimmungen erfolgten mit den Reagenzien und nach den Vorschriften der Firma Boehringer, Mannheim. Die Angaben der Enzymaktivitäten erfolgten in internationalen Einheiten $=\mu \mathrm{Mol}$ Substratumsatz pro Min.

\section{Ergebnisse}

Der Einbau von Leucin-[1-1.1 C] in das Protein der Rattenleber wird durch $\mathrm{CCl}_{4}$ schon sehr bald gehemmt. In der Zeit $30 \mathrm{M}$ lin. bis $60 \mathrm{M}$ lin. nach der Injektion von $\mathrm{CCl}_{4}$ ist die Einbaurate der Aminosäure gegenüber den Kontrollen hoch signifikant um 36\% erniedrigt (Tab. 1). Da gleichzeitig die säurelösliche ${ }^{14} \mathrm{C}$-Aktivität des nicht eingebauten freien Leucins signifikant ansteigt, dürfte es sich mit ziemlicher Sicherheit um eine Hemmung der Proteinsynthese handeln, zumal allein aus zeitlichen Gründen kein Anhalt für einen Isotopenverdünnungseffekt besteht. Die Hemmung der Proteinsynthese wird noch stärker, 2 Stdn. nach der $\mathrm{CCl}_{4}$-Injektion beträgt sie $45 \%$. Anders verhält sich die RNA-Synthese der Leber. Hier konnten wir mit der angegebenen Methodik keine signifikanten Veränderungen feststellen, insbesondere war der Einbau von mit Tritium markierter Orotsäure nicht vermindert. Der Protein-, RNA- und DNA-Gehalt, letzterer von uns als möglicherweise stabiles Bezugssystem gedacht, bleiben ebenfalls innerhalb der ersten zwei Stunden nach Tetrachlorkohlenstoft konstant.

Während der Inkubation einer normalen Rattenleber bei $37^{\circ}$ in feuchter Kammer nimmt die Aktivität der verschiedenen von uns untersuchten Enzyme zu. Das ist sowohl bei Extraktion des Gewebes mit Wasser der Fall (Tab. 2), als bei Verwendung von 0,1 M Phosphatpuffer als Homogenatmedium (Abb. 1). Bei der Extraktion der Leber mit Wasser macht sich besonders

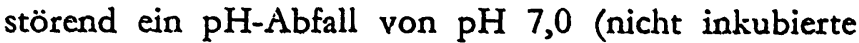
Lebern) auf etwa pH 6,0 (nach 6stdg. Inkubation) bemerkbar. Die Folge davon ist eine Abnahme der löslichen Proteine um 36\% nach $6 \mathrm{Stdn}$. Inkubation. Bezieht man die Enzymaktivität auf den Gehalt an löslichem Protein (Tab. 2b), so ist bei Extraktion mit Wasser auch die Malatdehydrogenase signifikant angestiegen mit einem Maximum nach $5 \mathrm{Stdn}$. Das Maximum der Lactatdehydrogenase nach $4 \mathrm{Stdn}$. ist auch dann gegenüber den Kontrollen signifikant verschieden, wenn man den Proteinabfall bei Extraktion mit Wasser nicht berücksichtigt (Tab. 2a). Bei der Extraktion der Leber mit 0,1M Na-Phosphatpuffer, pH 7,5 nimmt der Proteingehalt nach 6 Stdn. Inkubation nur um 9\% ab.
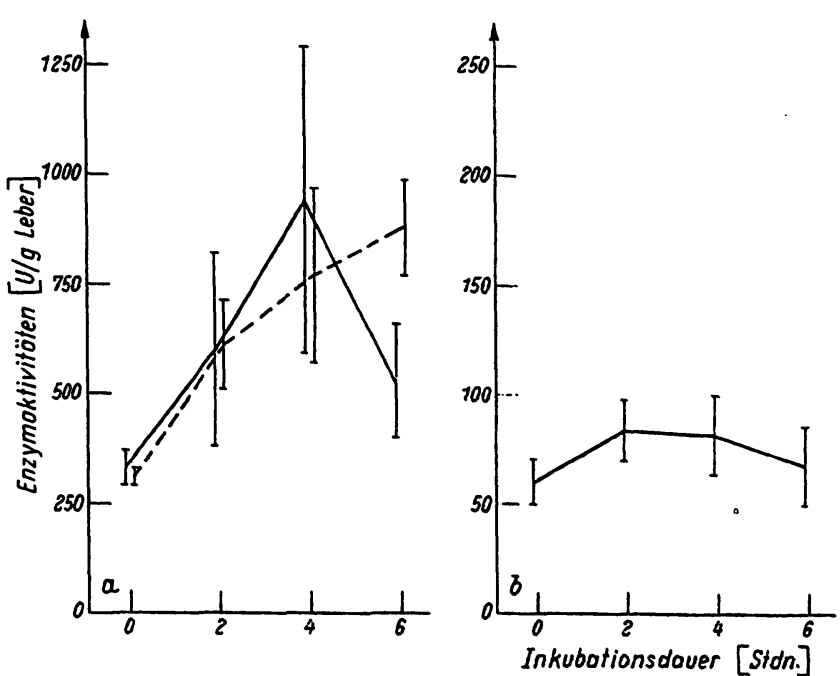

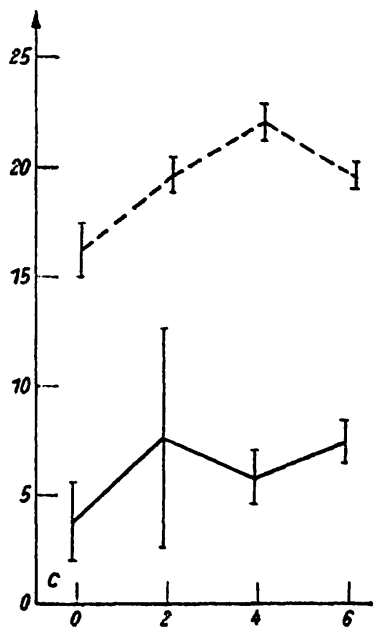

Abb. 1

Enzymaktivitäten nach Inkubation von Rattenlebern bei $37^{\circ}$ in vitro,

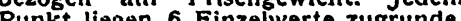
eingezeichnet ist die Standardabweichung

a) - Lactatdehydrogenase - Malatdeliyarogen

b) - - Aspartattransaminase

c) -_ Olutamatdehydrogenase -..-. Sorbitdehydrogenase 
Tab. 2 a

Aktivitätsänderungen verschiedener Leberenzyme sowie Gehalt an löslichem Protein nach Inkubation von Rattenlebern bei $37^{\circ}$ in feuchter Kammer. Gewebsextraktion mit bidest. Wasser. Enzymaktivitäten in IE ( $\mu$ Mol Substratumsatz pro Min.) und lösliches Protein in $\mathrm{mg}$ bezogen auf Leberfrischgewicht. Mittelwerte von je 6 Einzelwerten \pm Standardabweichung

\begin{tabular}{cccc}
\hline $\begin{array}{c}\text { Inkubations- } \\
\text { zeit [Stdn.] }\end{array}$ & \multicolumn{2}{c}{$\begin{array}{c}\text { Enzymaktivitäten in IE/g Leber } \\
\text { LHD }\end{array}$} & $\begin{array}{c}\text { Lösl. Protein } \\
\text { [mg/g Leber] }\end{array}$ \\
\hline Kontrollen & $454 \pm 118$ & $771 \pm 349$ & $143,4 \pm 8,7$ \\
1 & $902 \pm 77$ & $822 \pm 328$ & $113,2 \pm 4,1$ \\
2 & $1577 \pm 1445$ & $1031 \pm 283$ & $96,1 \pm 13,4$ \\
3 & $2370 \pm 2300$ & $936 \pm 343$ & $93,8 \pm 3,5$ \\
4 & $3287 \pm 199 * *$ & $802 \pm 288$ & $93,2 \pm 6,1$ \\
5 & $2842 \pm 1640$ & $1121 \pm 439$ & $95,2 \pm 8,5$ \\
6 & $1393 \pm 848$ & $966 \pm 278$ & $91,5 \pm 5,5$ \\
\hline
\end{tabular}

$* p<0,05 ; * * p<0,01$.

Tab. 2b Aktivitätsänderungen von Leberenzymen nach Inkubation von bezogen auf wasserlösliches Protein. Sonst wie in Tabelle $2 \mathrm{a}$

\begin{tabular}{ccc}
\hline $\begin{array}{c}\text { Inkubations- } \\
\text { zeit [Stdn.] }\end{array}$ & $\begin{array}{c}\text { Enzymaktivitäten in IE/g lösl. Protein } \\
\text { LDH }\end{array}$ & \multicolumn{1}{c}{ MDH } \\
\hline Kontrollen & $3180 \pm 805$ & $5380 \pm 2370$ \\
1 & $7960 \pm 675$ & $7260 \pm 2870$ \\
2 & $16670 \pm 14550$ & $10690 \pm 2540$ \\
3 & $25280 \pm 24300$ & $10050 \pm 3840$ \\
4 & $35630 \pm 22500 *$ & $8600 \pm 3330$ \\
5 & $29290 \pm 14100$ & $11760 \pm \pm 550 *$ \\
6 & $15150 \pm 9050$ & $10540 \pm 2890$ \\
\hline
\end{tabular}

$* p<0,05 ; * * p<0,01$.

Das Ausmaß des Aktivitätsanstieges bei den einzelnen Enzymen variiert gegenüber den bei Extraktion mit Wasser festgestellten, jedoch sind alle Maxima (Abb. 1) statistisch signifikant verschieden von den Kontrollen, und zwar: Lactatdehydrogenase $283 \% \mathrm{p}<0,01, \mathrm{Ma}-$ latdehydrogenase: $282 \% \mathrm{p}<0,001$, Aspartattransaminase: $142 \% \mathrm{p}<0,05$, Glutamatdehydrogenase: $200 \%$ $\mathrm{p}<0,02$ und Sorbitdehydrogenase: $134 \% \mathrm{p}<0,001$. Auch im Herzmuskel steigen während einer 4stdg. Inkubation in vitro bei $37^{\circ}$ die Enzymaktivitäten verschiedener Enzyme an (Abb. 2). Der Anstieg ist für die Malatdehydrogenase $(p<0,02)$ und die Aspartattransaminase $(p<0,05)$ statistisch signifikant, der Anstieg der Lactatdehydrogenase auf das mehr als

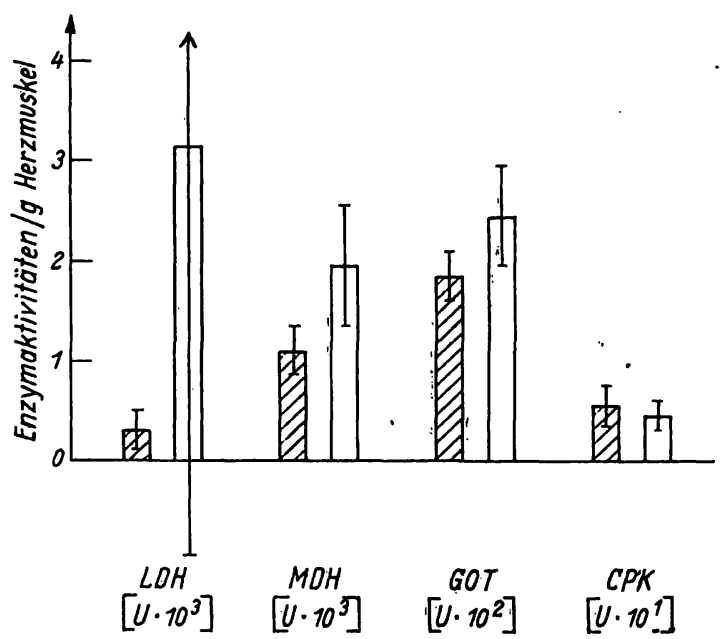

Abb. 2

Enzymaktivitäten im Herzmuskel normaler Ratten nach $4 \mathrm{Stdn}$ Inkubation in vitro bei $37^{\circ}$. Schraffiert: nicht inkubierte Kontrollen. Enzymaktivitäten von je 6 Einzelmessungen in $\mathrm{IE} / \mathrm{g}$ Frischgewicht zehnfache des Ausgangswertes ist nach dem t-Test nicht signifikant. An der Realität des Befundes hegen wir keinen Zweifel, zumal sich beide Kollektive nicht überschneiden und der niedrigste Wert des 4-Stdn.-Kollektivs noch außerhalb des Kontrollwertes + doppelte Standardabweichung liegt. Die Ausnahme bei den Enzymen des Herzmuskels bildet die Kreatinphosphokinase. Sie ist bekannt als relativ labiles Enzym und zeigt nach 4 Stdn. Inkubation einen Abfall ihrer Aktivität.

\section{Diskussion}

Eine Hemmung der Proteinsynthese in vivo, 3 bis $4 \mathrm{Stdn}$. nach Gaben von $\mathrm{CCl}_{4}$ ist von SMUCKLER, IsERI und BENDITT (9) beschrieben worden. Auf Grund unserer Enzymuntersuchungen (1) ließe sich vermuten, $\mathrm{da}$ der Hemmung der Proteinsynthese eine gesteigerte Proteinsynthese vorausginge. Das ist nicht der Fall, selbst in der von uns gewählten, relativ niedrigen Standarddosis, wird die Proteinsynthese der Leber in vivo schon in der ersten Stunde nach $\mathrm{CCl}_{4}$-Applikation gehemmt. Diese Hemmung der Proteinsynthese ist, wie wir zeigen konnten, nicht die Folge einer verminderten RNA-Synthese. SMUCKLER und Mitarbeiter (10) schließen aus ihren Befunden auf eine primäre Veränderung an den Mikrosomen als Ursache der $\mathrm{CCl}_{4}$-Schädigung. McLEAN und McLEAN (11) konnten zeigen, daß die Toxizität von Tetrachlorkohlenstoff wesentlich vom Funktionszustand der Mikrosomen abhängt. $\mathrm{Ob}$ dabei die Metabolisierung von $\mathrm{CCl}_{4} \mathrm{zu}$ einem toxischen Intermediärprodukt (12) durch vorausgehende eiweißfreie Ernährung gehemmt und durch Gabe von DDT (1.1.1-Trichloro-2.2-bis-( $p$-chlorophenyl-äthan) gesteigert wird (11), erscheint zweifelhaft. Diese Vorgänge müßten in unseren Versuchen in 30. Min ablaufen. Als morphologisches Äquivalent ist eine Vakuolenbildung des endoplasmatischen Reticulums mit einem Verlust von Ribosomen als erste Veränderung der subzellulären Strukturen der Leber 1 Std. nach $\mathrm{Gabe}$ von $\mathrm{CCl}_{4}$ beschrieben worden (13).

Die Hemmung der Proteinsynthese ist nicht nur als erste nachweisbare Tetrachlorkohlenstoffwirkung interessant. Sie macht es nahezu unwahrscheinlich, daß der früher von uns beobachtete Anstieg von Leberenzymen 2-4 Stdn. nach Gabe von $\mathrm{CCl}_{4}$ (1) auf einer gesteigerten Neusynthese von Enzymprotein beruht. Nun gibt es einen Zustand, bei dem man eine Aufhebung der Proteinsynthese wohl postulieren darf, der aber andererseits zu einem Anstieg von Serumenzymen führt, die Ischämie eines Organs. Unsere Beobachtungen bei der $\mathrm{CCl}_{4}$-Vergiftung ließen es nicht mehr unmöglich erscheinen, auch im ischämischen Organ einen Anstieg von Enzymen nachzuweisen. In der Tat findet sich sowohl in der ischämischen Leber als auch im ischämischen Herzmuskel ein Anstieg jener Enzyme, die später im Serum vermehrt nachzuweisen sind (Tab. 2, Abb. 1 und 2).

Die Ursache für diese Enzymaktivitätszunahme im Organ ist uns noch unbekannt. Wir möchten sie in 
Zusammenhang bringen mit Beobachtungen von JoHN und MrLler, die noch 2 Stdn. nach einer Hemmung des ${ }^{14} \mathrm{C}$-Einbaues in das Leberprotein durch Puromycin einen Anstieg von Serumalbumin und Fibrinogen im Perfusionsmedium fanden. Schmidt und Schmidr (15) registrierten einen Anstieg von Enzymaktivitäten in der isoliert perfundierten Leber nach Gabe von KCN. Auch die Beobachtungen von KENNEY (16) einer „Stabilisierung" der Tyrosintransaminase nach Hemmung der Proteinsynthese könnte auf einer „Freisetzung“ früher synthetisierten Enzymproteins beruhen.
Auf jeden Fall darf man wohl annehmen, daß irgendwelche „Strukturveränderungen" bereits vorhandener Proteine die Ursachen der Enzymaktivitätszunahme im geschädigten Gewebe sind, $\mathrm{da}$ eine Proteinzunahme durch Neusynthese ja ausscheidet. Im Hinblick auf den Mechanismus des Anstieges der Serumenzymaktivitäten nach Gewebsschädigungen bedeutet diese Erkenntnis zumindest eine Ergänzung der bisherigen Vorstellungen.

Wir danken Fräulein URsura GNEIsr für fleißige und gewissenhafte Mitarbeit bei der Durchführung der Versuche.

\section{Literatur}

1. KröNER, H und W. StaIB, diese Z. 6, 318 (1968). - 2. Bruns, F. und J. Neuraus, Arch. Biochem. Biophysics 55, 588 (1955). 3. KRÖNER, H. und W. StaIB, Hoppe Seyler's Z. physiol. Chem. 348, 575 (1967). - 4. Schimid, E., F. W. Schamid, H. D. HoRN und U. GerLaCH, in Methoden der enzymatischen Analyse, Hrsg. H. U. Bergmeyer Verlag Chemie, Weinheim/Bergstraße (1962). 5. KRÖNER, H. und W. StaIB, Acta cndocr. K'hvn 59, 193 (1968). 6. Werchselbaun, T. E., Amer. J. Clin. Pathol. 16, 40 (1946). 7. BuRton, K., Biochem. J. 62, 315 (1956). - 8. KRÖNER, H., B. Gutenberger, S. Hollahan und W. Starb, diese Z. 7, 8 (1969). - 9. Syuckler, E. A., O. A. Iseri und E. P. BenditT, Biochem. Biophys. Res. Comm. 5, 270 (1961). - 10. SMUCKLER,
E. A., B. Parthrer und T. Hultin, Biochem. J. 107, 151 (1968). 11. McLean, A. E. M. und E. K. McLean, Biochem. J. 100, 564 (1966). - 12. Rubinstenn, D. und L. Kanics, Canad. J. Biochem. 42, 1577 (1964). - 13. Stenner, G. W., M. J. Phillits und K. Mryar, in Internat. Review of Experimental Pathology Vol. 3. Hrsg. G. W. Richter und M. A. Epstein, Academic Press, New York (1964). - 14. JoHI, D. W. und L. L. MILLER, J. biol. Chemistry 241, 4817 (1966). - 15. Schmidr, E. in Stoffwechsel der isoliert perfundierten Leber, 3. Konferenz der Gesellschaft $f$. Biol. Chemie, Hrsg. W. Staib und R. Scholz, Springer, Berlin, Heidelberg, New York (1968). - 16. KenNex, F. T. Science, Washington 156, 525 (1967).
Prof. Dr. W. Staib 4000 Düsseldorf 1 Witzelstraße 111 